\title{
TOWARDS HOLISTIC ENERGY-EFFICIENT VEHICLE PRODUCT SYSTEM DESIGN: THE CASE FOR A PENALIZED CONTINUOUS END-OF-LIFE MODEL IN THE LIFE CYCLE ENERGY OPTIMISATION METHODOLOGY
}

\author{
Bouchouireb, Hamza (1); O'Reilly, Ciarán J. (1); Göransson, Peter (1); Schöggl, Josef-Peter (2); \\ Baumgartner, Rupert J. (2); Potting, José (1)
}

1: KTH Royal Institute of Technology, Sweden; 2: University of Graz, Austria

\begin{abstract}
The Life Cycle Energy Optimisation (LCEO) methodology aims at finding a design solution that uses a minimum amount of cumulative energy demand over the different phases of the vehicle's life cycle, while complying with a set of functional constraints. This effectively balances trade-offs, and therewith avoids sub-optimal shifting between the energy demand for the cradle-to-production of materials, operation of the vehicle, and end-of-life phases. The present work describes the extension of the LCEO methodology to perform holistic product system optimisation. The constrained design of an automotive component and the design of a subset of the processes which are applied to it during its life cycle are simultaneously optimised to achieve a minimal product system life cycle energy. A subset of the processes of the end-of-life phase of a vehicle's roof are modeled through a continuous formulation. The roof is modeled as a sandwich structure with its design variables being the material compositions and the thicknesses of the different layers. The results show the applicability of the LCEO methodology to product system design and the use of penalization to ensure solution feasibility.
\end{abstract}

Keywords: Design for X (DfX), Optimisation, Design methodology, Life cycle energy optimisation, Automotive product system design

Contact:

Bouchouireb, Hamza

KTH Royal Institute of Technology

The Centre for ECO2 Vehicle Design, AVE

Sweden

hamzab@kth.se

Cite this article: Bouchouireb, H., O’Reilly, C.J., Göransson, P., Schöggl, J.-P., Baumgartner, R.J., Potting, J. (2019)

'Towards Holistic Energy-Efficient Vehicle Product System Design: The Case for a Penalized Continuous End-of-Life Model in the Life Cycle Energy Optimisation Methodology', in Proceedings of the 22nd International Conference on Engineering Design (ICED19), Delft, The Netherlands, 5-8 August 2019. DOI:10.1017/dsi.2019.297 


\section{INTRODUCTION}

Energy-efficient vehicle concepts are a promising approach to curb the ever increasing energy demands of the transport sector. This sector represents just under 30\% of the total energy consumption of the European Union (EU) (European Commission, 2016). The transport energy needs of the EU are projected to grow by 0.4 to $1.4 \%$ (Ribeiro et al., 2007) while the EU-wide green house gas emissions are targeted to be reduced by at least $80 \%$ below the 1990 baseline levels by 2050 (European Climate Foundation, 2010). This trend increases the difficulty of meeting the challenge of modern vehicle design, which is to simultaneously meet the transport needs of society while minimising energy use and its associated environmental impact.

The Life Cycle Energy Optimisation (LCEO) methodology (O'Reilly et al., 2016) is a framework which is intended to enable early-stage energy-efficient vehicle design through finding the optimal design that minimises the total life cycle energy demand over the different phases of the vehicle's life cycle. In practice, the methodology is formulated as a mathematical optimisation problem, where the objective function to minimise is the life cycle energy of a vehicle, while the functional requirements which the design is subject to are implemented as mathematical constraints. The aforementioned energy acts as a life cycle environmental proxy allowing for the inclusion of environmental considerations early during the design process. This approach avoids the sub-optimal shifting of burdens between the energy demand for the cradle-to-production of materials, operation of the vehicle, and End-Of-Life (EOL) phases. Furthermore, in simultaneously considering the environmental and the technical aspects, the LCEO methodology allows the identification of critical trade-offs and enables the emergence of novel energy-efficient vehicle designs.

Previous works investigated the impact of the inclusion of the energies of different phases of the life cycle in the LCEO methodology. O'Reilly et al. (2016) investigated the impact of the inclusion of the production and use phase energies, while Jank et al. (2017) extended the framework through the additional consideration of the energy originating from the EOL phase. The latter was achieved through the inclusion of a closed-loop EOL model.

Within the context of the LCEO methodology, the life cycle energy of a given design is not only dependent on its associated design variable values; it is also significantly affected by modelling choices made prior to performing the design optimisation. These modelling choices generally aim at allowing all the material flows inherent to the vehicle's life cycle, as well as the transportation requirements to which it is subject, to be represented in a consistent manner from an energy perspective. These choices include the preselection of production and EOL processes which are included in the vehicle product system. In practice, this results in a set of parameters, referred to here as process design parameters, which enable the conversion of the material flows to their associated energy burdens and credits at the level of the optimisation framework's objective function. In particular, a subset of these parameters is introduced while assigning embodied energies to the different candidate materials in order to estimate the cumulative energy needed for the cradle-to-production phase of the vehicle's life cycle. Similarly, the processes which are included in the EOL phase of the vehicle are also explicitly fixed by dictating the amount of energy assigned as credit or burden to the system as a result of the application of an EOL process to the constitutive materials.

Thus, the optimal life cycle energy design resulting from this approach is optimal with respect to the preselected processes included in the vehicle product system. Specifically, the resulting design is optimal with respect to the process design parameters which result from the modelling of the processes that are included in the vehicle product system. Subsequently, a potential way of alleviating this dependency is to treat the process design parameters - or a subset of these parameters - as variables, referred to here as Process Design Variables (PDVs). Such an approach is synonymous with simultaneously performing the optimisation of the vehicle design and the process design. This could be argued to lead to life cycle energy optimal vehicle product systems.

However, this expansion of the design range of the LCEO methodology hinges upon the availability of an adequate representation of the PDVs. Namely, this representation can be discrete or continuous.

The first approach can originate from providing an exhaustive set of all the possible values that the design variable of a particular process can take based on existing or assumed processes. This representation has the drawback of leading to a Mixed Integer Non-Linear Problem (MINLP) formulation (Biegler, 2010), where a subset of the variables is continuous (e.g. a component's thickness) while 
the other is a set of discrete points. Despite the existence of solution methods for these formulations (Grossmann, 2002), they are typically associated with a significant potential increase in computational costs compared to their continuous variable counterparts (Kraemer et al., 2007). This increase is, in essence, due to the need of solving the continuous optimisation problem numerous times for different set combinations of the discrete variables in order to solve the larger mixed optimisation problem.

Alternatively, the PDVs can be continuously represented. They can be treated in a similar fashion to how a typical design variable, such as the volume fraction of a material or the thickness of a component, would be allowed to vary continuously within certain boundaries. This representation, when feasible, would result in a computationally tractable LCEO problem formulation. Nevertheless, allowing the PDVs to vary freely within too large boundaries might result in unfeasible solutions; and, per contra, setting too narrow boundaries might significantly reduce the exploratory ability of the LCEO methodology and subsequently hinder its potential to identify unconventional vehicle product system designs.

Thus, an attempt at preserving the exploratory ability of the LCEO methodology can be made through associating an energy "cost" to the PDVs. This continuous function would associate an assumed energy value to the PDV values, and it would be appended to the total life cycle energy of a given vehicle product system design. Subsequently, the optimiser would balance the individual energies stemming from the three main phases of the vehicle's life cycle with the energy stemming from the PDVs, and would result in an life cycle energy optimal vehicle product system.

The aim of this work is to establish how the LCEO methodology can be extended to enable holistic life cycle energy-efficient vehicle product system design. This is achieved by performing the life cycle energy design optimisation of a vehicle component product system while representing a subset of the processes that are employed during its EOL phase through continuous PDVs. As a consequence of this categorisation, the resulting design variables associated with the optimal life cycle energy design are both the vehicle sub-system design variables, as well as the process design variables associated with the EOL phase of the vehicle component. In particular, this approach is applied to the illustrative case of the design of a car roof sandwich panel product system under functional requirements. The design variables of the panel, which consist of the material compositions and thicknesses of the different layers, are simultaneously optimised with the process design variables of the EOL model. The latter phase is modelled through the substitution with a correction factor (van der Harst et al., 2016) approach within an assumed closed-loop recycling model. In this model the correction factors, which can be assumed to represent the ability of a recycling process to produce materials with virgin-like mechanical properties, are taken as the PDVs.

The novelty of this work resides in the extension of the existing discrete EOL model (Jank et al., 2017) to a continuous one, which allows for the concurrent optimisation of the vehicle design variables and the process design variables. This extension is enabled by the introduction of the continuous functions which associate energy "costs" to the PDV values. The core rationale behind their introduction as well as their impact on the resulting designs constitutes the major contribution of this work.

For the sake of clarity and self containment of the present work, section 2 will provide an overview of the LCEO methodology and the discrete EOL phase modelling as introduced in (O'Reilly et al., 2016) and (Jank et al., 2017). Section 3 will present the continuous EOL modelling and how it is implemented within the methodology. Section 4 will present the illustrative case study considered in this work as well as the results of the holistic product system life cycle energy optimisation for two different correction factor-energy relationship functions. The first approach is to assume that the correction factor values have an associated energy cost of 0 , and are effectively set to freely vary between their prescribed extremal values. The second approach is to assume that the correction factor-energy relationship is modelled as a logarithmic function. Section 5 examines the validity of the results through the vernacular of this study's enabling assumptions. In conclusion, future improvement avenues and applications for the continuous PDV augmented LCEO framework are identified.

\section{THE LIFE CYCLE ENERGY OPTIMISATION METHODOLOGY}

The LCEO method formally integrates environmental considerations into a design methodology through the formulation of a mathematical multidisciplinary design optimisation framework. The life cycle energy is used as the objective function to be minimised, as detailed in (O'Reilly et al., 2016). The 
functional requirements stemming from structural mechanics, and eventually other disciplinary fields, act as constraints on the design. Thus, this methodology does not compromise design requirements, but rather changes design variable values so as to find the design solution which features the minimum life cycle energy use while fulfilling all the transport-related functional requirements. The life cycle energy is formulated as

$$
E_{L}(X)=E_{P}(X)+E_{U}(X)+E_{E}(X),
$$

where $E_{L}$ is the life cycle energy, $E_{P}$ is the production energy, $E_{U}$ is the use-phase energy, $E_{E}$ is the end-of-life energy, and $X$ is the set of design variables. The production energy is obtained from the embodied energy of the constitutive materials of a given design, while the EOL energy is obtained from the energy credit or burden associated with subjecting the constitutive materials to different EOL processes. Finally, the use phase energy is obtained through multiplying the energy required to move the vehicle according to a prescribed drive cycle (New European Drive Cycle) by the number of such cycles during the entire use phase of a vehicle. The associated optimisation problem is expressed as

$$
\min \left(E_{L}(X)\right),
$$

subject to constraints of the form:

$$
\begin{array}{r}
T_{(I)}(X) \leq 0, \\
T_{(E)}(X)=0, \\
X_{\min } \leq X \leq X_{\max } .
\end{array}
$$

Equation 3 corresponds to functional requirements expressed as inequalities (I), while Equation 4 refers to the functional requirements which are expressed as equalities (E). The last equation, Equation 5, is the set of boundaries for the design variables.

The EOL phase of the vehicle's life cycle has been included in the framework through the modelling of recycling processes within an assumed closed-loop model. In particular, the substitution with a correction factor approach (van der Harst et al., 2016) has been adopted within an assumed closed-loop recycling model to avoid the system boundary expansions inherent to open-loop models. Within this attributional perspective, the recycled material is assumed to partially or completely substitute the input material; the substitution quota is accounted for through a correction factor. This modelling reflects the assumed practice of using recycled material to produce the same product as the one from which the recycled material originates. However, depending on the employed recycling methodologies, material properties can be degraded, leading to a partial substitution that is supplemented with the production of virgin material destined to the same end-product. The analysed product system incurs the burden for the EOL processing of the discarded analysed product, but also receives corrected credits for the amount of recycled material that is obtained from the EOL recycling of said analysed product. For instance, a correction factor of 0.2 , would imply that only $20 \%$ of the virgin energy production would be avoided when recycled materials re-enter the vehicle sub-system production system. Equivalently, the correction factor would reflect an assumed $80 \%$ degradation in material properties. As a result of this assumed technical property loss, it would be necessary to mix recyclates with virgin produced material, at $20 \%$ and $80 \%$ ratios respectively, in order to produce a product with high enough technical properties to meet the functional requirements constraining the design.

\section{CONTINUOUS EOL MODELING}

Previous work (Jank et al., 2017) included the identification of recycling technologies that are applicable to the candidate materials. These technologies were used to generate a set of scenarios combining different, yet fixed, correction factor values within a closed-loop model identical to the one introduced in the previous section. In that case, the correction factors were considered as process design parameters. In the present work, these coefficients are considered continuous PDVs. this alternative perspective requires an extension of the LCEO framework. As turning optimisation parameters into optimisation variables is accompanied by a need to define their bounds as well as develop an initial understanding of their potential impact on the life cycle energy objective function, and thereby the very behaviour of the whole optimisation framework. 
Firstly, the boundaries for the continuous correction factor PDVs can be derived from their definition (c.f. Section 2). From an ideal closed-loop perspective, a recycling technology would yield a recyclate with material properties similar to that of the virgin produced material, as a result of no process induced degradation. Thus, the maximal value for the correction factor is naturally set to 1 . It reflects the case where $100 \%$ of the recyclate can re-enter the production system and avoid $100 \%$ of the virgin energy production. By extension of the same reasoning to the worst case scenario, the minimum possible value for these continuous PDVs is set to zero.

Secondly, the total cumulative life cycle energy is a strictly decreasing function of the continuous correction factor PDVs. This behaviour derives from the role of the correction factor. It directly dictates the amount of energy that is credited back to the product system, while it does not have any direct bearing on the cradle-to-production energy and the operational phase energy. In other words, an increase of the value of the correction factor translates into a reduction of the perceived energy cost associated with the materials which figure in a given design. Hence, it can be deduced that it is highly likely that when the correction factors are set to freely and continuously vary between their extremal values, the optimisation process would result in their optimal value being their maximal allowed one.

However, the maximal physical PDV values are not always feasible ones. This is particularly relevant in the case of fibre reinforced plastics, where the recycling processes tend to shorten the fibres or damage their surfaces (Pickering, 2006). These two effects result in degraded material properties.

One way to enforce solution feasibility in the practice of constrained optimisation is to use penalty barrier functions (Wright and Nocedal, 1999). These are functions of the optimisation variables which are appended to the objective function. Their value increases to infinity as the candidate point for the optimisation approaches the boundary of the feasible region of the problem.

In this illustrative study, the feasibility of the solution of the PDV augmented LCEO formulation is enforced by modelling the relationship between the correction factors and their associated energy penalty through a logarithmic barrier function (Griva et al., 2009) of the form:

$$
g_{n}(x)=-\log \left(1-x^{n}\right), \quad \forall x \in\left[0,1\left[\text { and } \forall n \in \mathbb{N}^{*},\right.\right.
$$

where $g_{n}(x)$ is the penalisation coefficient associated to a given penalisation parameter $n$ and a given correction factor value $x$. Figure 1 shows the value of the penalisation coefficient associated with the logarithmic barrier function for different values of the penalisation parameter $n$. It can be observed that an increase in the value of $n$ results in a reduced penalisation of the relatively higher correction factor values. In other words, the penalisation coefficient controls the level of access to the higher correction values that is given to the optimiser. It is possible to tune the value of $n$ in order to render the correction factor values, which are beyond a predefined limit, very prohibitive from an energy standpoint.

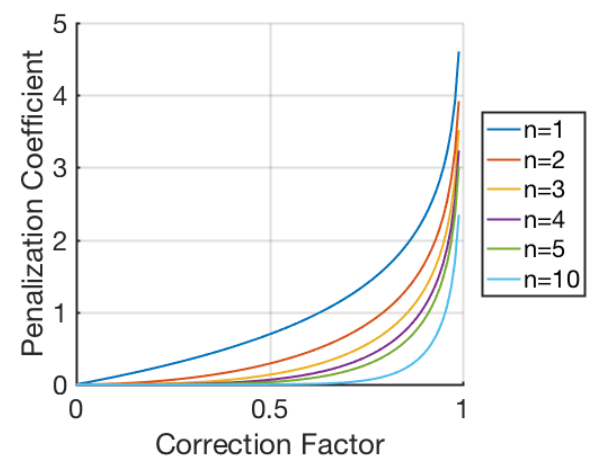

Figure 1. Value of the logarithmic barrier penalisation coefficient for different values of the correction factor and of the penalisation parameter.

A new penalised life cycle energy objective function is obtained from updating the previous life cycle energy objective function with the barrier functions:

$$
E_{P E N}=E_{P}(X)+E_{U}(X)+E_{E}(X)+\left|E_{E}(X)\right| \sum_{i=1}^{N_{m}} g_{n_{i}}\left(C_{f_{i}}\right),
$$


where $N_{m}$ is the number of design candidate materials to which a recycling process is applied, while $C_{f_{i}}$ is their associated correction factor and $n_{i}$ is their associated penalisation parameter. The penalisation functions are multiplied by the EOL energy in order to properly scale the penalisation energy (Griva et al., 2009). This is necessary in order to avoid a subset of the PDVs having an undue influence on the search process, or the lack of any influence at all. Furthermore, the EOL energy is taken in its absolute value to enforce the positivity of the scaling energy, since crediting the system is expressed through negative energy values. Such values would incentivise the optimisation in the wrong direction.

\section{RESULTS}

This section will briefly introduce the vehicle component product system on which the PDV augmented LCEO formulation will be applied. The main focus will be on the resulting designs for two different correction factor-energy relationship function choices. The first choice concerns the free optimisation case, where the correction factors have no associated energy burden. The second case features the modelling of the correction factor-energy relationship through a logarithmic penalisation function in order to ensure the feasibility of the resulting product system.

\subsection{Case study: the car roof sandwich panel}

The LCEO methodology is applied to the case study of the design of a vehicle component product system in order to illustrate its ability to perform holistic energy-efficient vehicle product system design. In particular, the vehicle component considered is the roof of a car. Such a component has already served as a benchmark for previous LCEO studies (O'Reilly et al., 2016; Jank et al., 2017). In this work, the roof is modelled as a 2D sandwich panel measuring $0.6 \mathrm{~m}$ of length.

Figure 2 lists the different design variables introduced by the sandwich panel structural choice: layer thicknesses $t_{i}$, as well as the material volume fractions $V_{i, j}$. The face sheets of the panel may be composed of a blend of Carbon Fibre (CF) and Glass Fibre (GF) as reinforcements to an epoxy matrix. The core of the panel can be composed of polyvinylchloride (PVC), polyethylene (PET) or polyurethane (PUR). The material properties of these material design variables, as well as their related production phase energies, are given in (O'Reilly et al., 2016). Note that the concept of hybridisation is used to allow for the continuous representation of the intra-layer material properties (Ashby and Bréchet, 2003). The Young's Modulus, density and Poisson's ratio are proportionally determined depending on the volume fractions, $V_{i, j}$, selected.

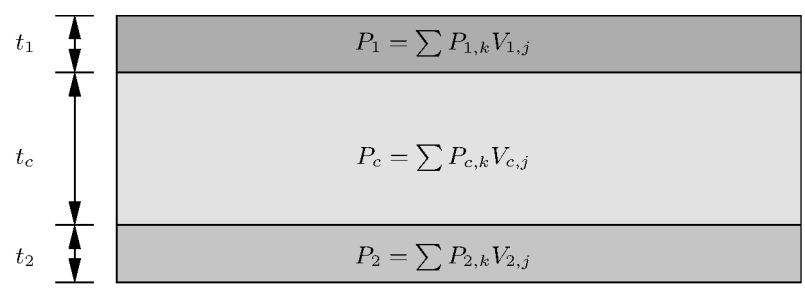

Figure 2. The sandwich structural choice dependent design variables.

The functional requirements which are constraining the design are of a similar nature to the ones formulates in (O'Reilly et al., 2016). The design is subject to two maximum displacement functional requirements set to $d_{1, \max }=d_{2, \max }=2.5 \times 10^{-6} \mathrm{~m}$. The former acts as a constraint on the acceptable load response to a localised static pressure, while the latter refers to the constraint on the acceptable load response to a static pressure distributed over the entire top of the panel. Two supplementary requirements are set for the vibrational behaviour of the panel. Minimum frequency constraints of $f_{1, \min }=330 \mathrm{~Hz}$ and $f_{2, \min }=520 \mathrm{~Hz}$ are respectively set for the first and second natural frequencies of the panel.

In the following, the processes which are assumed to be included in the EOL phase of the roof panel are the incineration with energy recovery of the core layer, CF chemical recycling as well as the mechanical grinding of GF. The energy credits (negative values) and burdens (positive values) associated with the application of these processes to the different constitutive materials are summarised in Table 1 . The correction factors which are associated with the processes applied to the CF and GF candidate materials are modelled as continuous PDVs. Thus, $N_{m}=2$ and $C_{f_{1}}$ and $n_{1}$ are respectively the correction factor and penalisation parameter associated with $\mathrm{CF}$, while $C_{f_{2}}$ and $n_{2}$ are the ones associated with the GF 
Table 1. The energy credits and burdens incurred by the vehicle product system for the EOL processing of the different candidate materials. Taken from (Jank et al., 2017)

\begin{tabular}{lllll}
\hline Carbon Fibre & Glass Fibre & Polyethylene & Polyvinylchloride & Polyurethane \\
\hline $38 \mathrm{MJ} / \mathrm{kg}$ & $0.17 \mathrm{MJ} / \mathrm{kg}$ & $-23.8 \mathrm{MJ} / \mathrm{kg}$ & $-22.9 \mathrm{MJ} / \mathrm{kg}$ & $-24 \mathrm{MJ} / \mathrm{kg}$ \\
\hline
\end{tabular}

material choice. The following results are obtained for a 60,000 km drive cycle. The first minimum eigenfrequency requirement is the active constraint limiting any further minimisation of the life cycle energy in all the following resulting designs.

\subsection{LCE optimisation in the free continuous PDV case}

The resulting panel design variables and their associated optimal life cycle energy PDVs for the unpenalised continuous PDV optimisation case are presented in Table 2. The final life cycle energy design features a top sheet which is over two times thicker than the bottom one, $0.85 \mathrm{~mm}$ and $0.38 \mathrm{~mm}$ respectively, with a core thickness of $40 \mathrm{~mm}$. The core is also composed of a blend of the softer PUR with the stiffer PVC, at $37 \%$ and $63 \%$ ratios respectively, while the face sheets are solely composed of CF. Table

Table 2. Resulting optimal design variables and PDVs for the free optimisation case

\begin{tabular}{llllllllllll}
\hline$V_{1, C F}$ & $V_{1, G F}$ & $V_{2, C F}$ & $V_{2, G F}$ & $V_{c, P E T}$ & $V_{c, P V C}$ & $V_{c, P U R}$ & $t_{1}[\mathrm{~mm}]$ & $t_{2}[\mathrm{~mm}]$ & $t_{c}[\mathrm{~mm}]$ & $C_{f, C F}$ & $C_{f, G F}$ \\
\hline $100 \%$ & $0 \%$ & $100 \%$ & $0 \%$ & $0 \%$ & $63 \%$ & $37 \%$ & 0.85 & 0.38 & 40 & 0.99 & 0.47 \\
\hline
\end{tabular}

3 presents the individual energies associated with the different phases of the life cycle of the optimal design as well as its associated life cycle energy and total mass. The EOL energy associated with the life cycle energy optimal design balances, for the most part, the embodied energy of the panel. The final design is also characterised by a $247.6 \mathrm{MJ}$ life cycle energy and weighs $1.096 \mathrm{~kg}$. These results

Table 3. Resulting energies and mass of the free optimisation case

\begin{tabular}{lllll}
\hline Production energy & Use energy & EOL energy & Life cycle energy & Total mass \\
\hline $145.5 \mathrm{MJ}$ & $224.1 \mathrm{MJ}$ & $-122 \mathrm{MJ}$ & $247.6 \mathrm{MJ}$ & $1.096 \mathrm{~kg}$ \\
\hline
\end{tabular}

confirm the analysis performed in Section 3. Namely, the CF correction factor PDV takes the maximal allowable value, thus making the CF material choice for the face sheets even more attractive from the optimiser's perspective. This is due to the amalgam of CF's relatively superior material properties with the possibility offered by the unpenalised formulation to alleviate its high embodied energy. In fact, by setting $C_{f_{1}}$ to virtually 1 , the system is significantly credited for an intensive use of CF in the resulting design. This is reflected by the face sheets being solely composed of CF.

The resulting $C_{f_{2}}$ is set to 0.47 . It is worth noting that this value does not carry any particular significance - for this particular optimal design, in this formulation — as GF does not enter into the material composition of the final design. This value stems from the use of a derivative-free heuristic optimiser (Price et al., 2006) to solve the energy minimisation problem. A randomly generated population of candidate design solutions is evolved and mutated over a predetermined number of iterations and the member of the final population with the minimum life cycle energy value is selected as the optimal solution. In this particular case, the latter member carried that particular value of $C_{f_{2}}$. Performing the optimisation multiple times yields the same life cycle energy optimal design variables, with the same value of the CF recycling PDV, but with different values for the GF recycling PDV.

\subsection{LCE optimisation in the penalised continuous PDV case}

Figure 3 shows the results of the life cycle energy optimisation for the logarithmically penalised continuous PDVs case. The results are shown for a single CF penalisation parameter, $n_{1}=1$, while the GF penalisation parameter, $n_{2}$, is varied from 1 to 10 . The following quantities are plotted against $n_{2}$, from left to right and top to bottom: the EOL PDVs, the material compositions of the different layers, the face sheet thicknesses, the core layer thicknesses, the life cycle energy and its penalised counterpart as well as the resulting designs' masses.

It can be seen from the plot describing the evolution of the EOL PDVs as functions of the GF penalisation parameter that the penalisation parameter has the intended effect on the PDVs resulting from the 
optimisation process. Namely, an increase of the value, which represents a relaxation of the penalisation on the higher correction factor values, results in increased optimal values of the GF associated PDV. In particular, the optimal $C_{f_{2}}$ value increases from 0 to 0.51 . It is also worth noting that the variation of the degree of penalisation of $C_{f_{2}}$ results in a slightly decreasing $C_{f_{1}}$ over the span of the penalisation parameters, with its average value being 0.26 .

The increases in the value of $C_{f_{2}}$ have a direct impact on the presence of GF in the material composition of the life cycle energy optimal panel designs. The presence of GF in the bottom face sheet increases from $0 \%$ to $25 \%$ as the penalisation is relaxed from $n_{2}=1$ to 10 . The mix of GF and CF in the bottom layer results in a hybrid-material (Ashby and Bréchet, 2003) based sheet with a decreased stiffness in comparison to a sheet made up solely of CF and with an identical thickness. This decrease in stiffness is balanced by an increase of the thickness of the top facing sheet, which is solely composed of CF.
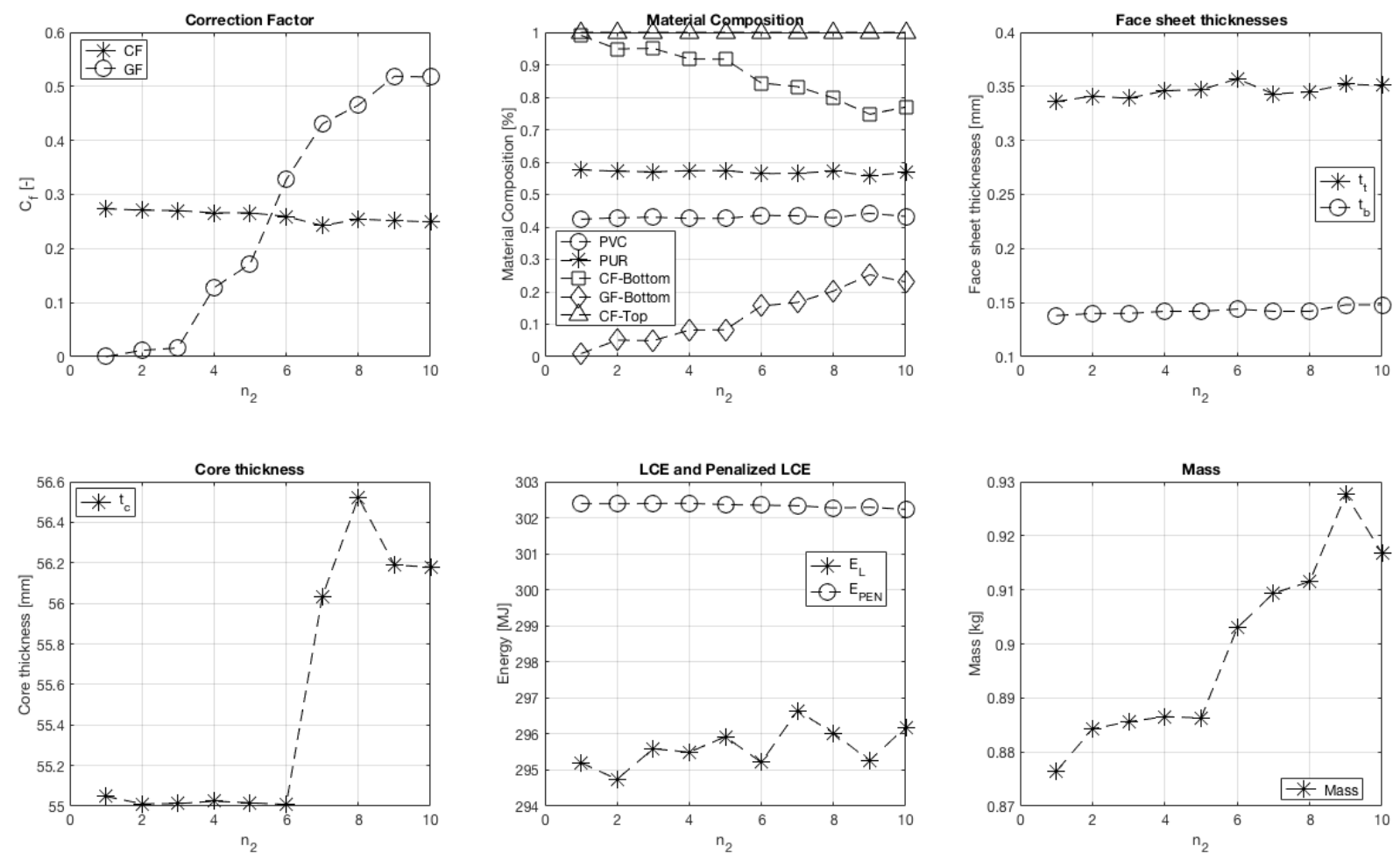

Figure 3. Results of the continuous penalised PDV case. The following quantities are plotted against $n_{2}$, from left to right and top to bottom: the EOL PDVs, the layers' material compositions, the face sheet thicknesses, the core layer thicknesses, the life cycle energy and the penalised one as well as the resulting designs' masses. $n_{2}$ is varied from 1 to 10 .

However, changing the thickness of the top layer is not the only degree of freedom at the optimiser's disposal to maintain the overall stiffness of the design at the functionally required level. In fact, when the penalisation is relaxed from $n_{2}=6$ to 7 , the thickness of the top sheet decreases from 0.357 to 0.343 $\mathrm{mm}$ while the thickness of the core increases. The latter was largely left unaffected by the incremental relaxations of the penalisation of the GF associated PDV up to, and including, $n_{2}=6$. It subsequently increases by an average of $1.2 \mathrm{~mm}$ up to $n_{2}=10$. However, the resulting material composition of the core is kept unchanged. This indicates that the overall structural stiffness is maintained by increasing the geometrical stiffness of the structure through increasing the thickness of its core layer. The optimiser, which earlier relied on increasing the amount of $\mathrm{CF}$ in the top facing sheet to preserve the overall structural stiffness, relies subsequently on the main feature of sandwich structures (Zenkert, 1995): increasing the bending stiffness through an increased distance between the two load-carrying face sheets.

The penalised life cycle energy is slightly decreasing with the increase of the parameter $n_{2}$. This decrease is explained by the successive increases in the optimal value of the GF associated PDV, which dictates that the system is increasingly credited for employing GF in its face sheets. The effect of the more significant presence of GF in the resulting designs can all also be noticed in their masses. The mass tends 
to increase with increases in the penalisation parameter. Thus, under the assumptions of the penalised continuous PDV optimisation, heavier designs coincide with more energy-efficient product systems. This conclusion still holds when the results of the penalised model are compared with the free continuous optimisation case's. In fact the resulting design of Section 4.2 weighs $1.096 \mathrm{~kg}$, with an associated life cycle energy of $247.6 \mathrm{MJ}$, as opposed to just under $0.93 \mathrm{~kg}$, and about $295 \mathrm{MJ}$, for the heaviest penalised design. This is due to the impact of the logarithmic penalisation function. In the first case, the perceived energy cost of the use of CF is lower than the cost of capitalising on the inherent geometrical stiffness offered by the sandwich structure design choice. Since increasing the thickness of the core would increase the material flows associated with PVC and PUR, which are incinerated with energy recovery during the EOL phase of the vehicle component. Thus, the system is not credited as much as when employing thicker face sheets made of CF in conjunction with a high correction factor.

\section{DISCUSSION}

The previous results demonstrate the sensitivity of the designs resulting from the penalised continuous PDV augmented LCEO formulation to the choice of penalisation parameters. The choice has also been shown to directly impact which mechanisms drive the optimiser to the minimum life cycle energy design, namely material-choice related stiffness as opposed to geometrical stiffness.

The resulting designs are not only sensitive to the penalisation parameter choice, but also to the choice of the functions representing the PDV-energy relationship. These functions are not a priori known, and their choice constitutes an integral part of the modelling phase of the LCEO formulation. The relative complexity introduced by this additional modelling phase is outweighed by the flexibility it offers. Indeed, it potentially allows to perform LCEO studies with different intents.

Penalisation functions are adequate PDV-energy relationship candidate functions as they allow for the fine tuning of the feasibility of the resulting design. Additionally, as shown in Section 4.3, they also facilitate the identification of the underlying mechanisms which enable trade-offs between technical and environmental aspects. In doing so, they can potentially help the designer in managing the complexity of the design task and identify the vehicle sub-system's environmental criticalities more effectively.

Alternatively, for a given candidate material, all the available applicable processing technologies can be used as data points over which a PDV-energy relationship function can be interpolated. In constructing the functions in such a manner for all, or a select few, candidate materials, the resulting designs would not only be life cycle energy optimal vehicle sub-system designs, but would also contain their optimal PDVs, and by extension their associated optimal processing technologies. This approximation, when applicable, preserves the computational tractability of the original LCEO formulation.

The PDV augmented LCEO methodology allows to tackle two main barriers to the effective implementation of quantitative ecodesign tools in industrial environments (Rossi et al., 2016). Firstly, the methodology implicitly compares different product system configurations and provides designers with the best alternative while allowing for the identification of trade-off mechanisms in terms of environmental and technical aspects. Secondly, the PDV formulation helps designers maintain solution feasibility across an extended range of product characteristic configurations by taking into account different processes. To the knowledge of the authors, such flexibility is novel within the field of quantitative ecodesign methods and tools.

The novelty of the augmented LCEO formulation, coupled with its ability to tackle established barriers to the wider adoption of ecodesign tools, warrant further methodological developments. In particular, the robustness of the resulting designs to the PDV-energy relationship function choice should be further examined and guidelines for a standardised approach to constructing the relationship functions should be devised. Furthermore, the sensitivity of the resulting designs with respect to modelling uncertainties should be analysed and quantified.

\section{CONCLUSION}

This work has shown how the LCEO methodology can be extended to perform holistic product system optimisation through simultaneously performing vehicle sub-system design optimisation, as well as process design optimisation. This extension was based on considering previous parameters of the optimisation as continuous process design variables, and the introduction of continuous functions which map the different possible values of these variables to energy burdens. Applying this approach to the 
illustrative case study of the design of a car roof sandwich panel resulted in the formulation of a continuous EOL model. The inclusion of the latter in the optimisation framework has shown the ability of the augmented formulation to offer finer control of the feasibility of the resulting holistic design. Furthermore, the potential of the methodology to identify the underlying mechanisms enabling trade-offs amongst the component's technical aspects was demonstrated. Finally, the sensitivity of the resulting designs to the choice of process design variable-energy relationship functions implies that further work is needed to define and formulate these mapping functions.

\section{REFERENCES}

Ashby, M. and Bréchet, Y. “Designing hybrid materials”, Acta materialia, Vol. 51 No. 19, pp. 5801-5821, 2003. https://doi.org/10.1016/s1359-6454(03)00441-5

Biegler, L. Nonlinear Programming. Society for Industrial and Applied Mathematics, 2010. https://doi.org/10.1137/1.9780898719383

European Commission. EU Transport in figures. Publications Office of the European Union, 2016.

European Climate Foundation. Roadmap 2050; a practical guide to a prosperous, low-carbon europe. 2010.

Griva, I., Nash, S. G. and Sofer, A. Linear and nonlinear optimization, volume 108. Siam, 2009. https://doi.org/10.1137/1.9780898717730

Grossmann, I. E. "Review of nonlinear mixed-integer and disjunctive programming techniques", Optimization and Engineering, Vol. 3 No. 3, pp. 227-252, Sep 2002. https://doi.org/10.1002/cite.201400037

Jank, M.-H., O’Reilly, C. J., Göransson, P., Baumgartner, R. J., Schöggl, J.-P. and Potting, J. “Advancing energy efficient early-stage vehicle design through inclusion of end-of-life phase in the life cycle energy optimisation methodology". In Ecological Vehicles and Renewable Energies (EVER), 2017 Twelfth International Conference on, pp. 1-9. IEEE, 2017. https://doi.org/10.1109/ever.2017.7935888

Kraemer, K., Kossack, S. and Marquardt, W. "An efficient solution method for the minlp optimization of chemical processes", Computer Aided Chemical Engineering, Vol. 24 No. 105, 2007. https://doi.org/10.1016/s1570-7946(07)80041-1

Luttropp, C. and Lagerstedt, J. "Ecodesign and the ten golden rules: generic advice for merging environmental aspects into product development”, Journal of Cleaner Production, Vol. 14 No. 15, pp. 1396-1408, 2006. https://doi.org/10.1016/j.jclepro.2005.11.022

O’Reilly, C. J., Göransson, P., Funazaki, A., Suzuki, T., Edlund, S., Gunnarsson, C., Lundow, J.-O., Cerin, P., Cameron, C. J., Wennhage, P. et al. "Life cycle energy optimisation: A proposed methodology for integrating environmental considerations early in the vehicle engineering design process", Journal of Cleaner Production, Vol. 135, pp. 750-759, 2016. https://doi.org/10.1016/j.jclepro.2016.06.163

Pickering, S. J. "Recycling technologies for thermoset composite materials current status", Composites Part A: applied science and manufacturing, Vol. 37, pp. 1206-1215, 2006. https://doi.org/10.1016/j.compositesa.2005.05.030

Price, K., Storn, R. M. and Lampinen, J. A. Differential evolution: a practical approach to global optimization. Springer Science \& Business Media, 2006. https://doi.org/10.1007/3-540-31306-0_13

Ribeiro, S. K., Kobayashi, S., Beuthe, M., Gasca, J., Greene, D., Lee, D. S., Muromachi, Y., Newton, P. J., Plotkin, S., Sperling, D. et al. Transportation and its infrastructure. 2007.

Rossi, M., Germani, M. and Zamagni, A. "Review of ecodesign methods and tools. Barriers and strategies for an effective implementation in industrial companies", Journal of Cleaner Production, Vol. 129, pp. 361-373, 2016. https://doi.org/10.1016/j.jclepro.2016.04.051

van der Harst, E., Potting, J. and Kroeze, C. "Comparison of different methods to include recycling in lcas of aluminium cans and disposable polystyrene cups", Waste management, Vol. 48, pp. 565-583, 2016. https://doi.org/10.1016/j.wasman.2015.09.027

Wright, S. and Nocedal, J. "Numerical optimization”, Springer Science, Vol. 35 No. (67-68), p. 7, 1999. https://doi.org/10.1007/b98874

Zenkert, D. An introduction to sandwich structures. Student edition, 1995.

\section{ACKNOWLEDGMENTS}

The authors would like to thank the Centre for $\mathrm{ECO}^{2}$ Vehicle Design at KTH, funded by the Swedish Innovation Agency Vinnova, aimed at supporting the development of resource efficient vehicles in a sustainable society; and the strategic research area TRENoP and the Swedish Research Council Formas for their financial contributions to this work. 